University of Nebraska - Lincoln

DigitalCommons@University of Nebraska - Lincoln

Norman R. Simon Papers

Research Papers in Physics and Astronomy

$12-2002$

\title{
Source localization of MEG sleep spindles and the relation to sources of alpha band rhythms
}

llonka Manshanden

MEG-Centre KNAW, i.manshanden@vumc.nl

Jan C. De Munck

Vrije Universiteit Medical Center

Norman R. Simon

University of Nebraska - Lincoln, nsimon@unl.edu

Fernando H. Lopes da Silva

Dutch Epilepsy Clinics Foundation, f.h.lopesdasilva@uva.nl

Follow this and additional works at: https://digitalcommons.unl.edu/physicssimon

Manshanden, Ilonka; De Munck, Jan C.; Simon, Norman R.; and Lopes da Silva, Fernando H., "Source localization of MEG sleep spindles and the relation to sources of alpha band rhythms" (2002). Norman $R$. Simon Papers. 52.

https://digitalcommons.unl.edu/physicssimon/52

This Article is brought to you for free and open access by the Research Papers in Physics and Astronomy at DigitalCommons@University of Nebraska - Lincoln. It has been accepted for inclusion in Norman R. Simon Papers by an authorized administrator of DigitalCommons@University of Nebraska - Lincoln. 


\title{
Source localization of MEG sleep spindles and the relation to sources of alpha band rhythms
}

\author{
Ilonka Manshanden, ${ }^{1}$ Jan C. De Munck, ${ }^{1}$ Norman R. Simon, ${ }^{2}$ and Fernando H. Lopes da Silva ${ }^{3}$ \\ 1. MEG Center, Vrije Universiteit Medical Center, Polikliniek, receptie C, P.O. Box 7057, 1007 MB, Amsterdam, The Netherlands \\ 2. Department of Physics and Astronomy, University of Nebraska-Lincoln, Lincoln, NE, USA \\ 3. Dutch Epilepsy Clinics Foundation, Heemstede, The Netherlands
}

Corresponding author - I. Manshanden, tel 131-20-444-0722, fax 131-20-444-4816, email i.manshanden@vumc.nl

\begin{abstract}
Objective: First, to determine the distribution of the estimated sources of sleep spindles, and alpha and mu rhythms based on whole-head magnetoencephalogram (MEG) recordings; second, to scrutinize the physiological relevance of the dipole fit algorithm in localizing on-going normal rhythmic activities.

Methods: One hundred and fifty-one channels were used to record spontaneous MEG activity during wakefulness and superficial sleep in 4 normal subjects. The equivalent dipolar sources were estimated by a new "dipole fit algorithm" and projected on the corresponding magnetic resonance images.

Results: Equivalent dipoles of MEG spindles were distributed over the centro-parietal region. Those of alpha rhythms were concentrated around the occipito-parietal sulcus and those of mu rhythms were confined to the area around the central sulcus.

Conclusions: MEG sleep spindles, and alpha and mu rhythms have distinct spatial distributions of their equivalent dipolar sources. This demonstrates that various cortical regions that oscillate within the same frequency band have different spatial organizations and different functional aspects.
\end{abstract}

Keywords: sleep spindles, alpha rhythm, mu rhythm, magnetoencephalogram, electroencephalogram, source localization

\section{Introduction}

With the introduction of the magnetoencephalogram (MEG) especially when combined with magnetic resonance imaging (MRI), the search for the brain sources of sensory and motor evoked events received novel inputs that gave new vigor to human neurophysiology. Most of these studies focused on transient events such as epileptic spikes that are readily amenable for this kind of analysis. However, the study of on-going brain activity, namely brain rhythms, lagged behind due to the methodological difficulty of analyzing continuous signals of relatively long duration. For instance whether alpha and mu rhythms on the one hand, and sleep spindles on the other, are generated in distinct or overlapping cortical areas is still not clearly known. In order to solve this question advanced spatio-temporal analysis methods are necessary. The recent development of a new algorithm (De Munck et al., 2001b) with the aim of estimating sources of large datasets, as is the case with this kind of signals, led us to investigate whether generators of spontaneous MEG/ electroencephalogram (EEG) rhythms in the alpha band, namely sleep spindles, and the alpha and mu rhythms, are distributed over distinct or overlapping cortical areas.

The basic approach consists in finding good fitting dipoles using a dipole model applied on each time sample. First, the data is band-pass filtered to select the rhythm of interest, then a global search algorithm is applied. The equivalent dipoles encountered are then plotted on the corresponding MRI slice of the brain.

To the best of our knowledge no previous study based on MEG measurements has investigated the spatial relation of MEG spindle sources with the sources of the MEG alpha and mu rhythms in a comprehensive way. Nevertheless there are reports of the localization of alpha and mu rhythms, and sleep spindles, but taken separately. It is clear that there is a magnetic equivalent of the electrical sleep spindle (Hughes et al., 1976, Nakasato et al., 1990, Lu et al., 1992, Simon et al., 2000). Using combined MEG and EEG recordings Yoshida et al. (1996) proposed a model for 3 types of sleep spindles based on the appearance of sleep spindles in MEG or EEG alone, or simulta- 
neously in both MEG and EEG. Lu et al. (1992) measured sleep spindles with a 24-channel SQUID gradiometer and concluded that multiple generators were active during one sleep spindle train. Principal component analysis (PCA) revealed that 4-6 components were needed to explain $90 \%$ of field variance of $1 \mathrm{~s}$ long spindle. Furthermore a single dipole model and a moving two-dipole model could not accurately account for the underlying sources. Shih et al. (2000) performed multiple dipole modeling of $100 \mathrm{~ms}$ epochs of MEG sleep spindles. Using this model they found sources in all brain lobes. Regarding the source models of EEG sleep spindles the recent work of Anderer et al. (2001), using a distributed source model (low-resolution electromagnetic tomography, LORETA), demonstrated that simultaneously active cortical sources, differing in frequency, were located medially and bilaterally in the frontal lobe (frequency $<13 \mathrm{~Hz}$ ) and medially in the precuneus in the parietal lobe (frequency $>13 \mathrm{~Hz}$ ). It is important to compare source models with electrophysiological measurements obtained directly from the cortex. Caderas et al. (1982) observed in intracranial recordings sleep spindles at the frequency of $11-12 \mathrm{~Hz}$ in the frontal lobes. Nevertheless, the frontal sources of sleep spindles are still controversial since electrographic recordings obtained in epileptic patients with indwelling electrodes in the orbito-frontal cortex showed very little spindle activity in these cortical areas (Uchida et al., 1999).

Regarding source models of alpha rhythms several authors (Lu et al., 1992, Salmelin and Hari, 1994, Salenius et al., 1995, Hari et al., 1997) reported dipolar sources predominantly located in the parieto-occipital area, while additional dipoles were located around the calcarine fissure (Williamson and Kaufman, 1989, Salmelin and Hari, 1994, Salenius et al., 1995, Hari et al., 1997, Parra et al., 2000). With intracranial electrodes in the human brain, alpha rhythms could be traced throughout the whole occipital cortex (Perez-Borja et al., 1962). With respect to the mu rhythm there are source models showing dipolar sources located in the somatosensory cortex (Tiihonen et al., 1989, Salmelin and Hari, 1994, Hari et al., 1997) that correspond well to results of corticograhic recordings (Arroyo et al., 1993, Crone et al., 1998). Our objectives were to determine the distribution of the estimated sources of sleep spindles, and alpha and mu rhythms based on whole-head MEG (and EEG) recordings obtained from the same subjects by using a dipole fit algorithm which has the capacity to calculate sources of large amounts of data.

\section{Methods}

Sleep recordings of 4 healthy subjects were made; 3 males (aged 47, 34, and 38 years) and one female (aged 24 years). The MEG dewar was tilted to $75^{\circ}$ and the subjects were able to lie horizontally while recordings took place. MEG/EEG measurements were made inside a 3-layer magnetically shielded room (Vacuum Schmelze,
$\mathrm{GmbH}$, Germany). Sleep data were recorded until sufficient stage 2 non-rapid eye movement (NREM) sleep data were collected.

For EEG recordings electrodes were placed on the skull according to the international 10-20 system and recorded referential to the left earlobe. In subject 1 the EEG was recorded from 7 scalp positions, namely $\mathrm{Fpz}, \mathrm{Fz}, \mathrm{Cz}$, $\mathrm{Pz}, \mathrm{O} 2, \mathrm{C} 3$, and C4. For subjects 2 and 3 the 20 positions of the 10-20 system were used. To optimize EEG source localization, 64 electrodes were placed on the scalp of subject 4 according to the international 10-10 system. Electrode impedances were kept below $5 \mathrm{kOhm}$. The signals were amplified and digitized using the CTF hardware system. MEG recordings were made using a 151-channel whole-head MEG system (CTF Systems Inc., Vancouver), with sensors uniformly distributed on the helmet surface, mean spacing approximately $3.1 \mathrm{~cm}$. The pick-up coils are first-order axial gradiometers with $2 \mathrm{~cm}$ diameter and $5 \mathrm{~cm}$ baseline. An additional reference array was used for noise cancellation by means of software formation of second- and third-order synthetic gradiometers. The MEG signal was recorded in third gradient. Both MEG and EEG sleep signals were digitized at a sampling rate of $250 \mathrm{~Hz}$. A digital hardware anti-aliasing low-pass filter of $80 \mathrm{~Hz}$ was used and the powerline was eliminated with notch filters. An analog second order high-pass $0.16 \mathrm{~Hz}$ filter was applied to the EEG signals to reduce the effect of electrode potential shifts. Additionally, the left and right median nerves were stimulated to record the somatosensory evoked field (SEF) responses (sample rate $1250 \mathrm{~Hz}$, stimulus rate $2 \mathrm{~Hz}$, pulse duration $0.2 \mathrm{~ms}$ ). The source localization of the N20 response was used to determine the location of the central sulcus, which was in turn used as a topographic reference.

The analyzed sleep data consisted of epochs of $10 \mathrm{~min}$. Just before and after each epoch the position of the head relative to the MEG sensor array was monitored using 3 head localization coils. Epochs in which head movement exceeded $0.5 \mathrm{~cm}$ were discarded. The coils were placed on the left and right pre-auricular impressions and on the nasion. They were also used as fiducial markers for coregistration with MRIs.

High-resolution T1-weighted MRIs (86 contiguous sagittal slices, $2 \mathrm{~mm}$ thick) were obtained for each subject from a Siemens Magnetom Vision (1.5 T). To enable coregistration with MEG, vitamin E capsules were attached to the subject's head on the same points as the localization coils.

Sleep spindles were visually selected off-line from stage 2 NREM sleep (subject 1: 80 MEG and 81 EEG spindles; subject 2: 99 MEG and 93 EEG spindles; subject 3: 50 MEG and 74 EEG spindles; subject 4: 117 MEG and 118 EEG spindles). Additionally epochs of alpha and $\mathrm{mu}$ activity were selected in periods when the subject was awake and relaxed with eyes closed. MEG and EEG power spectra of the spindles, were computed from epochs which were defined from $1 \mathrm{~s}$ preceding the first spin- 
A

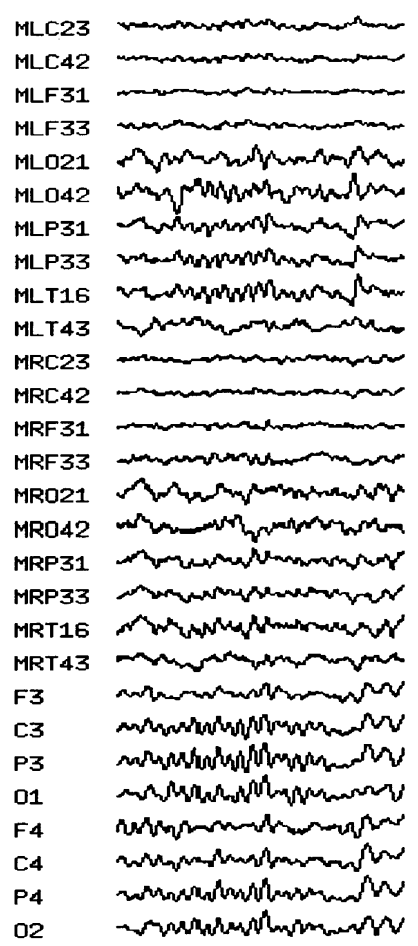

B

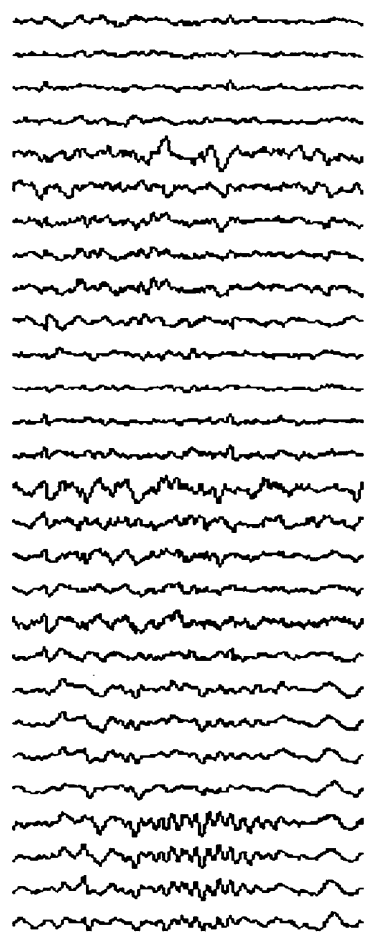

C

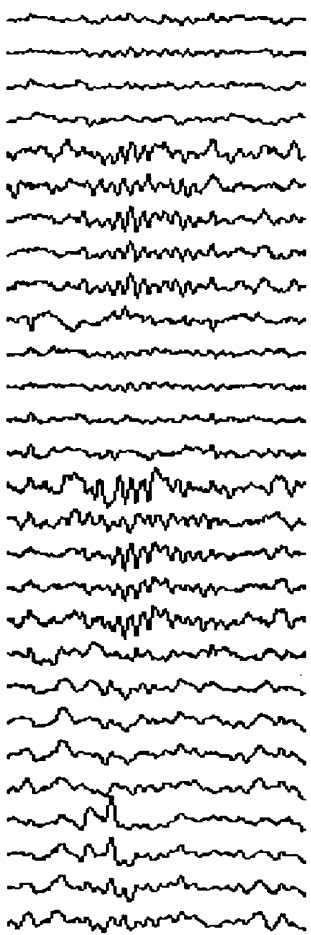

$3 \mathrm{pT}, 200 \mu \mathrm{V}$

Figure 1. Raw MEG (upper traces) and EEG (lower traces) data of 3 types of spindles of subject 2. (A) Spindle simultaneous in MEG and EEG. (B) Spindle only in EEG. (C) Spindle only in MEG.

dle sample point to $3 \mathrm{~s}$ after this sample point. A fast Fourier transform routine was applied to these $4 \mathrm{~s}$ epochs, which resulted in a frequency resolution of $0.25 \mathrm{~Hz}$. The fast Fourier transformed amplitudes from two consecutive data sets were averaged (under the constraint that the head positions of the two data sets coincided). Principal component analysis (PCA) of the band-pass filtered epochs between the beginning and end markers was carried out to calculate the number of statistically independent components contributing to a sleep spindle.

For source localization the data were band-pass filtered between 9 and $16 \mathrm{~Hz}$ (third order Butterworth filter) to enhance the signal to noise ratio. EEG data of subject 4 were converted to average reference. At each sample point within the selected spindles the source was calculated using an equivalent dipole source model in a spherical model of the head (i.e. a time-varying model). The position and radius of the sphere of each subject was optimized on the basis of the corresponding MRIs. To optimize the source localization of the EEG signal (data of subject 4) a 3-layered volume conductor model was applied. For each layer, representing the skin, the skull, and the brain, a different relative conductivity was used (1.0, 0.013, and 1.0, respectively). The coordinates of the 64 electrodes were determined with respect to the nasion-ear head coils. This was done using an ad- ditional coil through which a current was fed while it was pointed at the electrodes. The magnetic field of this coil was measured by the MEG device and used for localization of the coil (De Munck et al., 2001a). A sphere, fitted through the electrode positions on the head, was used as the outer sphere of the head model for the EEG source localization.

The optimal dipole positions and orientations were defined by using the least squares cost function. Local minima were avoided by using a new dipole fit algorithm (De Munck et al., 2001b) that uses a global search strategy. In the model the constraint was applied in such a way that two sources, one in each hemisphere, are symmetrical in space, whereas orientation and strength could vary between hemispheres (position symmetrical equivalent dipole model (PSD)). Only data points with a residual error lower than $10 \%$ were selected for further analysis. The dipoles were projected on the MRI of the subject using the image-fusion software package "ConQuest viewer" (Van Herk et al., 2000 and Hoogeman et al., 2000). Additionally the MR image was divided into voxels with a size of $1 \mathrm{~cm}^{3}$, which was a good compromise between spatial resolution and accuracy. The number of dipoles which fell within a given voxel, was counted. This number is displayed on the MRI slices that contain the corresponding voxel using a color code. 


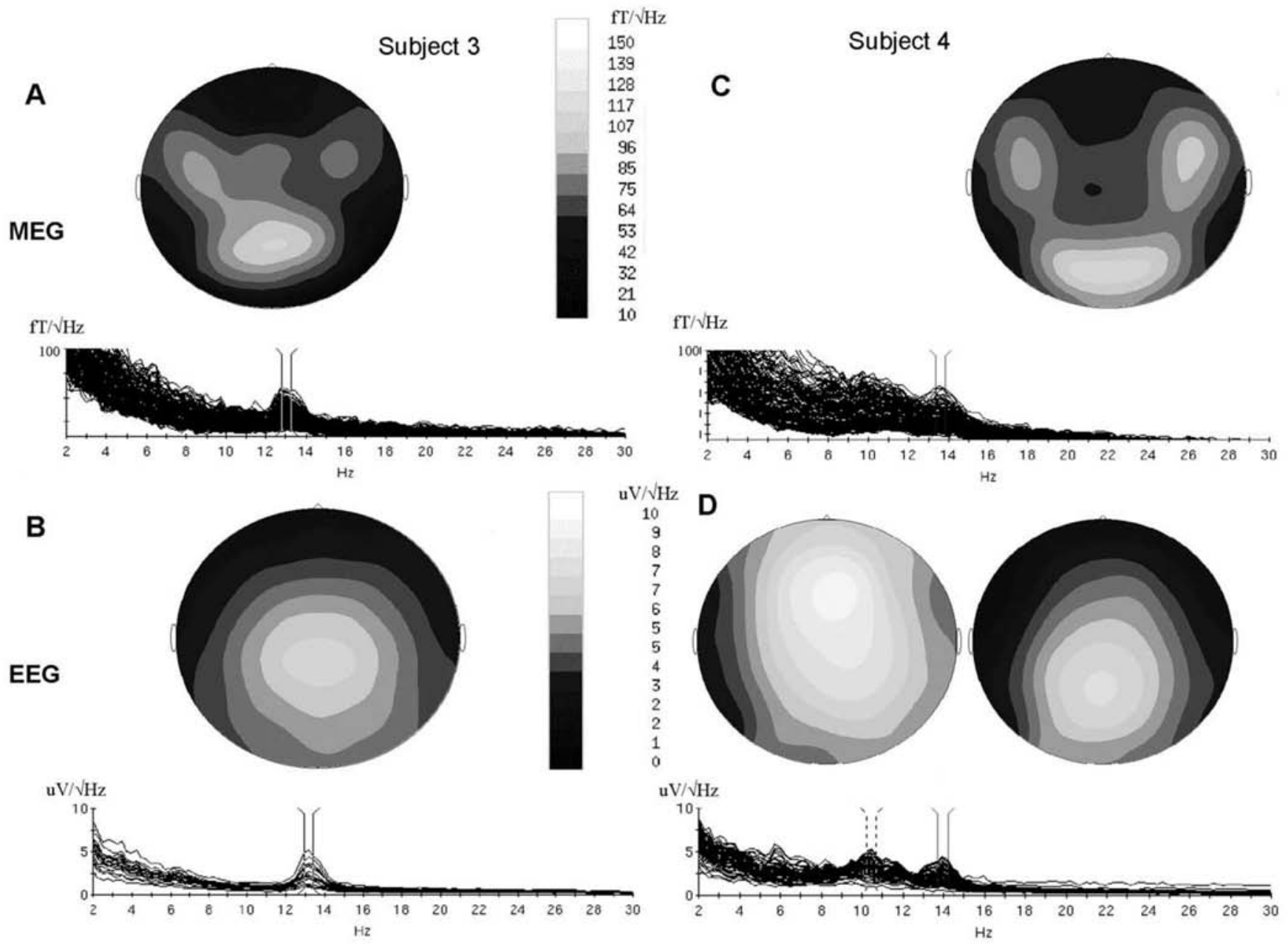

Figure 2. Power spectra of averaged $4 \mathrm{~s}$ spindle epochs of MEG and EEG of subjects 3 and 4 plotted together with the power distribution of the spindle peaks. The channels are superimposed. (A) MEG spectrum of subject 3. (B) EEG spectrum of subject 3 . (C) MEG spectrum of subject 4. (D) EEG spectrum of subject 4. All 4 spectra showed the "fast spindle" frequency (13-14 Hz) with maximal power in the posterior and temporal areas. Only the EEG spectrum of subject 4 (D) displayed a "slow spindle" peak $(10.5 \mathrm{~Hz})$ with an anterior distribution.

\section{Results}

In most cases spindle activity can be recorded simultaneously in MEG and EEG (Figure 1a), suggesting a tangential orientation of the spindle source(s). However, the EEG spindles were more pronounced than the MEG spindles. Less frequently, spindles appeared in EEG while they were not detectable in MEG (Figure 1b) which indicates the existence of more radially oriented sources in some cases. The occurrence of spindles in MEG only was also found (Figure 1c).

Power spectra of both MEG and EEG spindles were made to verify whether the subjects displayed a mono- or bimodal pattern. The results of subjects 3 and 4 are plotted in Figure 2together with the power distribution projected on a 2D-map. The spectra of all channels were superimposed for MEG and EEG separately. Both the MEG (Figure 2a) and the EEG (Figure 2b) spectra of subject 3 showed a clear peak in the "fast frequency spindle range"
(13.0 and 13.2, respectively). The MEG/EEG spectra of subjects 1 and 2 (data not shown) corresponded with that of subject 3 (subject 1: EEG $13 \mathrm{~Hz}$, MEG $13.5 \mathrm{~Hz}$; subject 2: EEG $14.5 \mathrm{~Hz}, \mathrm{MEG} 14.0 \mathrm{~Hz}$ ). A "slow frequency spindle peak" could not be distinguished in these subjects. The EEG power distribution in the $13 \mathrm{~Hz}$ band of subject 3 was maximal in the central, parietal, and occipital leads (Figure 2b), while the MEG power distribution was prominent in the posterior and temporal sensors (suggesting bilateral activity in the centro-parietal regions). Similar spatial MEG patterns were found for subjects 1 and 2 .

The peak frequency of the MEG spindle activity $(13.5 \mathrm{~Hz})$ of subject 4 and the corresponding spatial distribution (Figure 2c) were comparable to the other 3 subjects, although less distinct due to the presence of slower frequency activity in the spectrum of this subject. However, this activity did not seem to include a "slow frequency spindle peak" but merely a broad spectrum of slower brain activity. Even when the MEG channels were individ- 


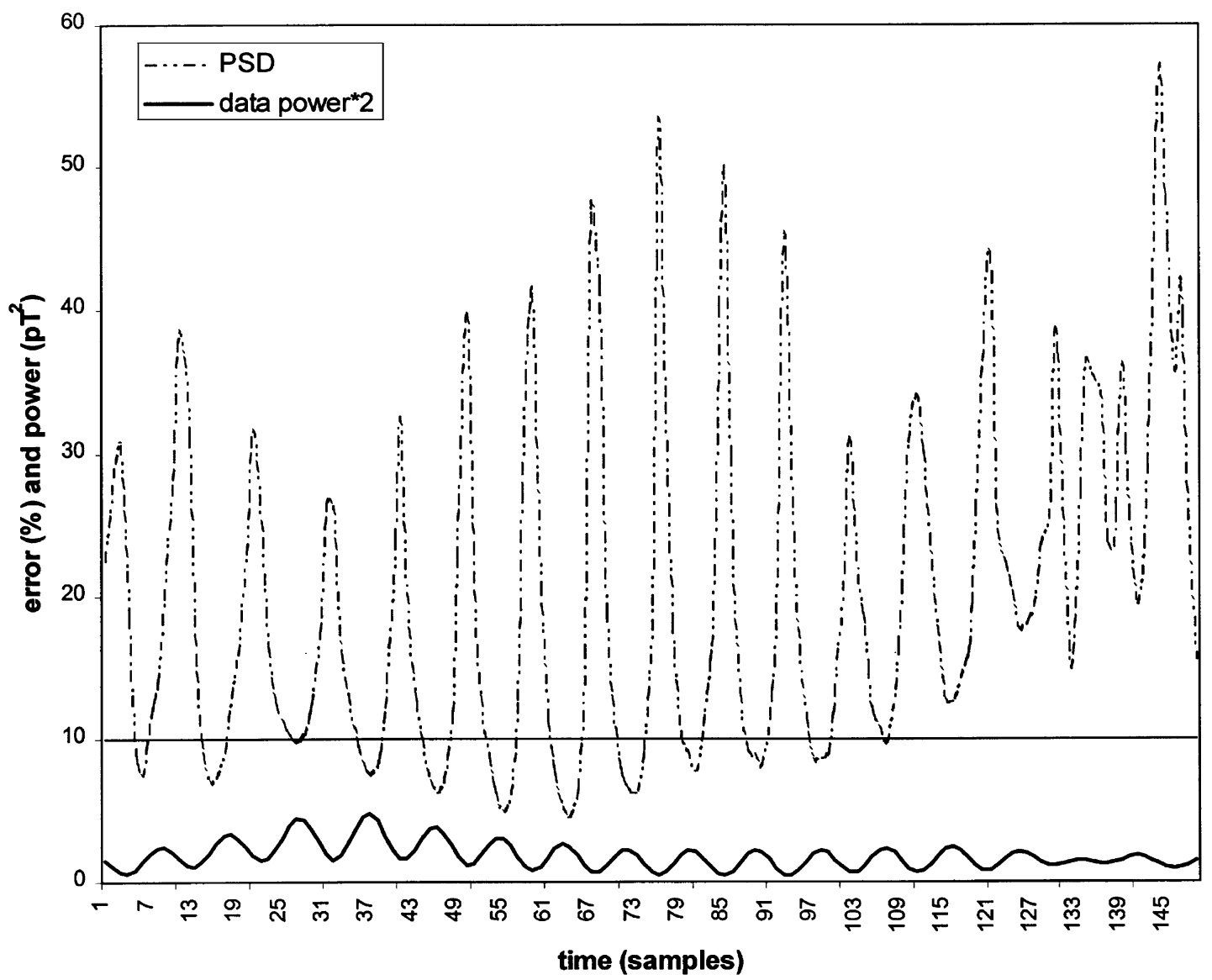

Figure 3. PCA of one spindle of subject 1 . The data was band-pass filtered (9-16 Hz) before PCA was applied. The two major components are plotted as a function of time. The major component (dense line) accounted for $30 \%$ of the signal, the second component (dotted line) accounted for $26 \%$ of the signal. Note the temporal variability of the two components.

ually inspected a "slow frequency spindle peak" was not discernible. The EEG spectrum of subject 4 (Figure 2d) displayed, in contrast to the EEG spectra of the other subjects and in contrast to the MEG spectra of all subjects studied, a bimodal spindle frequency with peak frequencies at 10.5 and $14 \mathrm{~Hz}$. Again the $14 \mathrm{~Hz}$ peak was most prominent in the central, parietal, and occipital leads, whereas the "slow frequency spindle peak" displayed a maximum in the midfrontal and midcentral leads.

PCA of the MEG spindles revealed that at least 5 components were needed to explain $90 \%$ of the field variance of the filtered spindle epochs. One example of the time functions of the two major components of one spindle is depicted in Figure 3. The contributions of the two components to the signal varied in time. The major component, which in this case accounted for $30 \%$ of the total field variance, was dominant in the second part of the spindle, whereas the second component $(26 \%)$ dominated the first part of the spindle. The time functions of the components displayed a sinusoidal behavior (which reflected the spindle waveform), with variable phase shifts between the components.

Figure 4shows the results of the time-varying PSD model, fitted on a spindle of subject 2 . The residual error is plotted as a function of time (sample points within the spindle); additionally the total field power of the filtered spindle is plotted. The $10 \%$ error level is marked as a solid line. In general, the performance of the model improved as the spindle power increased, whereas the residual error increased as the power of the signal diminished. However, in the first part of the spindle when the power was maximal, the residual error did not have the lowest values. Apparently, the behavior of the spindles is complex.

In Figure 5the spatial relations among dipoles of MEG spindles, and mu and alpha rhythms of the 4 subjects are plotted in 3 planes (sagittal, coronal, and axial). Wake recordings of subject 3 were done after the MRI acquisition. The dental fillings of this subject were magnetized due to the magnetic field of the MR apparatus, which introduced artifacts in the MEG signal. This made it impossible to perform reliable source localization of alpha and mu activity of this subject. Therefore only dipoles of the spindle activity of subject 3 are shown. In all subjects the dipoles of the MEG spindles (red dots) were restricted to the centro-parietal region of the brain, only the spindle dipoles of subject 2 spread somewhat more anteriorly compared to the other 3 subjects. Clusters of MEG alpha activity (blue dots) and mu activity (green dots) are most clearly discernable in subject 4 . The MEG alpha cluster was concentrated around the parieto-occipital sulcus, the 


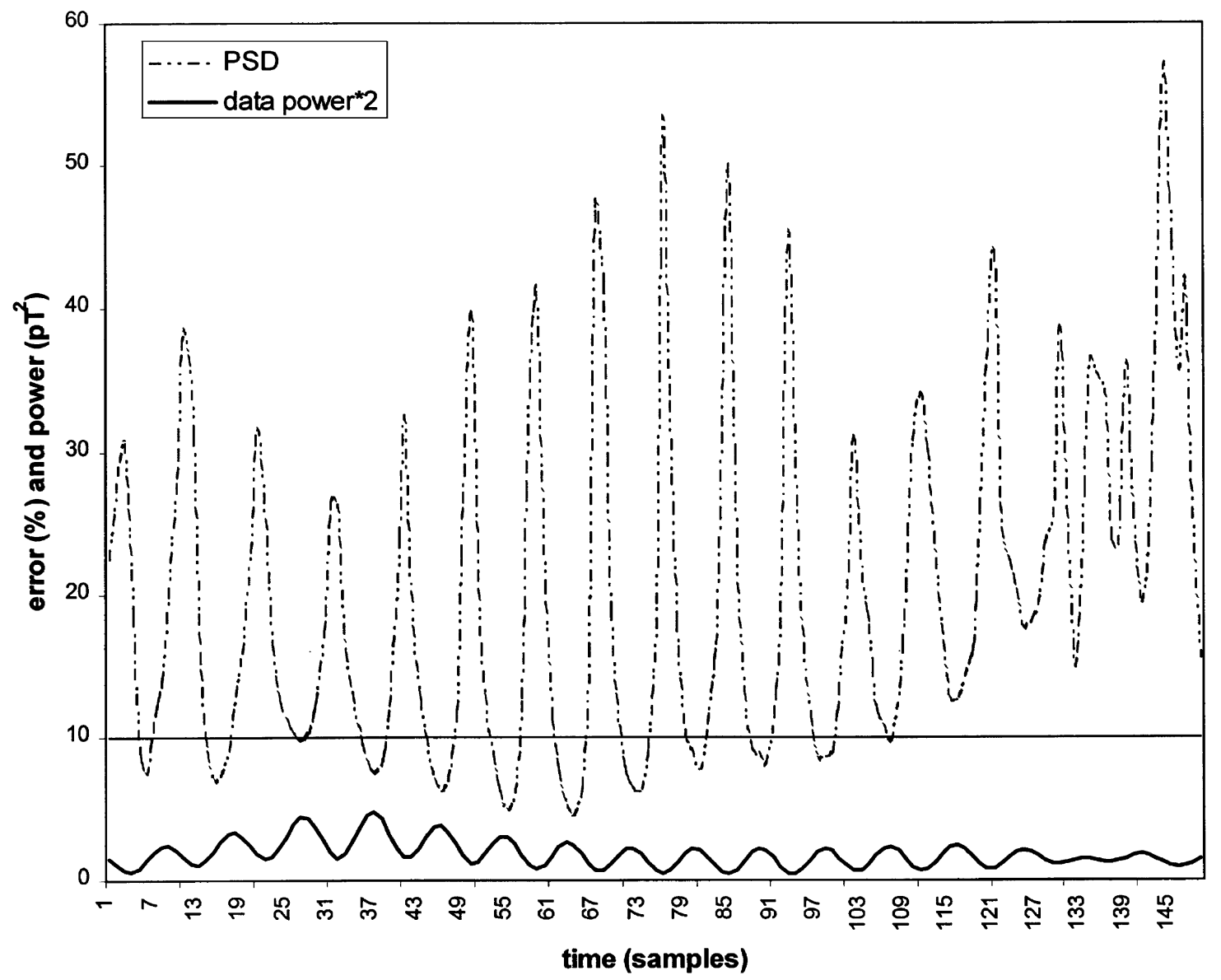

Figure 4. Time functions of the PSD (dashed line) model fitted on one spindle of subject 2, plotted together with the spindle power (thick line). The $10 \%$ error level is indicated as the horizontal solid line.

dipoles of the MEG mu rhythm were mainly restricted to the central sulcus (see also Figure 8). In subjects 1 and 2 there appeared to be a similar tendency for the behavior of the clusters of the mu and alpha rhythms, although these clusters were less circumscribed compared to those of subject 4 . In subjects 1 and 2 the dipoles of the MEG alpha rhythm adjoined the lower posterior part of the spindle dipoles, whereas the mu dipoles were found approximately in the middle-upper central region where spindle dipoles were also present.

In the previous plots the equivalent dipoles were projected on one single plane of a sagittal MRI slice. This is a convenient way to obtain a global view of the distributions of the sources of various brain rhythms of all 4 subjects, without using many plots. However, in this way the spatial gradient of the distribution of the dipoles on the other planes is not taken into account. This important information can be visualized by dividing the sphere into voxels and plotting the number of dipoles within each voxel on the corresponding MRI plane. This yields information about the density of the dipoles within each voxel and permits one to identify "hot spots" on the corresponding MRI slice. It also takes into account differences in numbers of dipole positions for each rhythm by normalizing the dipole distribution with respect to the max- imal number of dipoles present. The plots are referred to as dipole density plots. Furthermore these plots permit to obtain insight into the 3D distribution of the sources of spindles, and mu and alpha rhythms. This was achieved by plotting the dipole density plots on several equidistant sagittal MRI slices. Such dipole density plots of subject 4 are shown in Figure 6(MEG) and Figure 7(EEG). These figures demonstrate that the alpha and spindle dipoles appear in more medial MRI slices whereas the mu rhythm is visible in more lateral slices. The voxels with the highest number of dipoles (i.e. the "hot spots" in red) were located deeper for the spindles when compared to the red voxels of the alpha and mu rhythm. The figures demonstrate that the "hot spots" of the alpha rhythm for MEG and EEG were confined to the region of the parietooccipital sulcus. Figure 7a displays a limited number of voxels in the posterior part of the brain which contain a relatively high number of EEG spindle dipoles and voxels with a smaller number of dipoles in more frontal areas.

To verify whether the dipoles of the mu rhythm were indeed located near the central sulcus, the position of the source of the SEF after stimulation of the medial nerve was determined. In Figure 8the dipoles of the mu rhythm of the EEG (white dots) and MEG (black dots) of subject 4 were plotted in relation to the position of the N20 

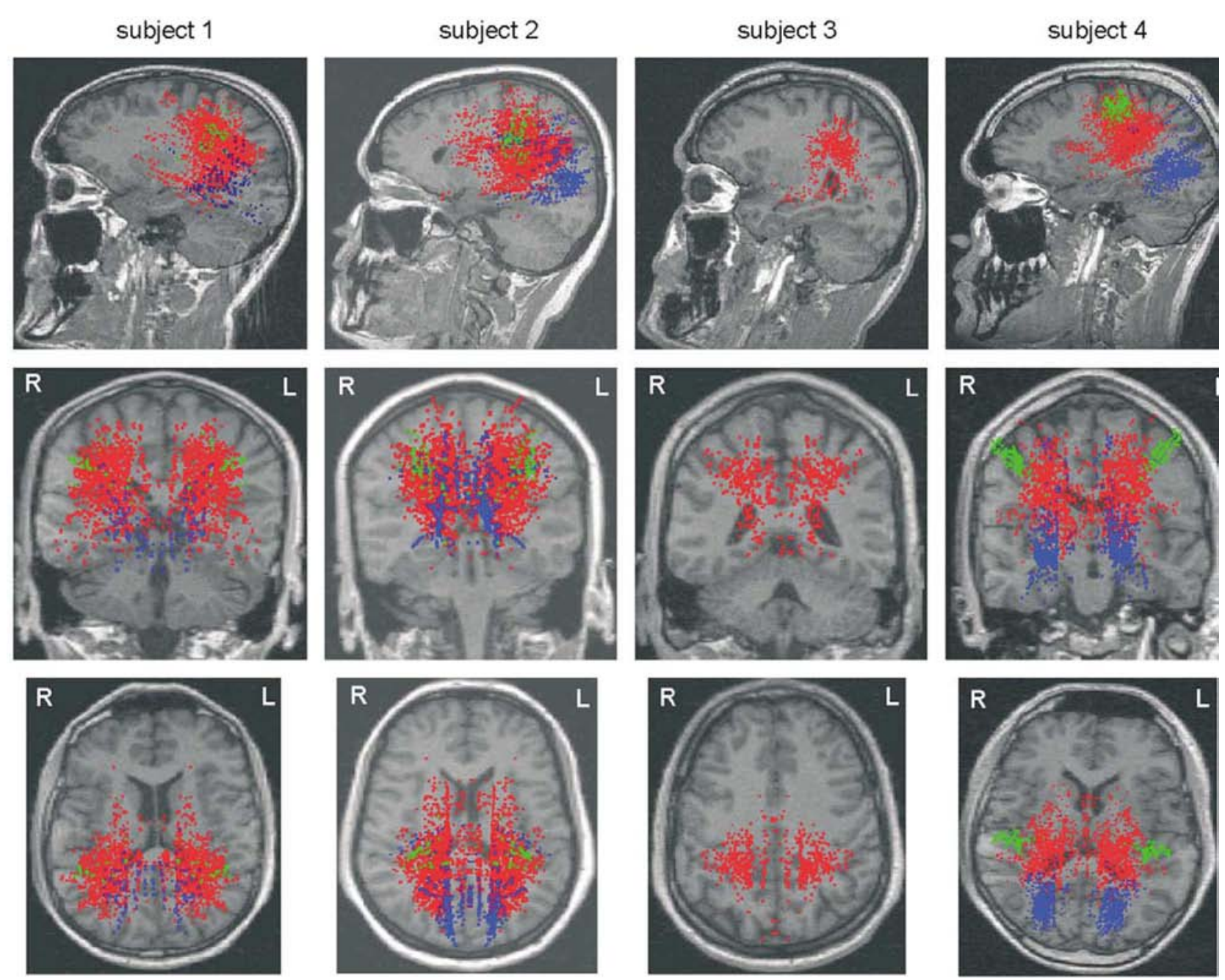

Figure 5. Comparison of dipole localizations of spindles, and alpha and mu rhythms in MEG of the 4 subjects (subject 3 only spindles) in 3 planes (sagittal, coronal, axial). Green dots, mu rhythm; blue dots, alpha rhythm; red dots, spindles. Spindle dipoles were found in the centro-parietal regions of all subjects. Mu dipoles were restricted to the central area, whereas dipoles of the alpha rhythm were situated in more posterior parts of the brain.

component of the SEF (large white dot). The MEG mu dipoles followed the course of the central sulcus and overlapped with the SEF source in the superficial part of the sulcus but extended along and around the central sulcus to deeper lying areas. EEG mu dipoles, however, were less restricted to the central sulcus. They were scattered around a larger area with a preference to more medial and deeper lying areas.

\section{Discussion}

First of all it is important to examine the meaning of equivalent dipolar sources of ongoing brain activity. This feature is often underexposed in articles on source localization. Sleep spindles, alpha rhythms, and to a lesser extent mu rhythms, are brain rhythms that can be recorded from several locations at the scalp. This indicates that extended cortical areas are involved in the generation of these signals. The use of equivalent dipoles as source models for these distributed brain activities there- fore yields an oversimplified solution to the problem of determining the underlying sources of these signals. The equivalent dipoles should be viewed just as descriptors of the "center of gravity" that best describe, in a statistical sense, the spatial distribution of the corresponding active cortical area at a given time. The position of the dipoles with respect to the cortical surface depends on the extent and geometry of the activated cortical area: superficially positioned dipoles (i.e. near to the cortical surface) like those of the mu rhythm, corresponding to more localized cortical activity, while deep lying dipoles, like those of sleep spindles, representing rather the activity of extended cortical surfaces. Thus dipole locations give only an approximation of the localization of the active brain area and the extension of the area. Nevertheless the approach followed here requires a small number of assumptions about the configuration of the sources as compared to alternative approaches. This was an important argument to choose this kind of model in order to compare the distribution of MEG sources of 3 types of spontane- 


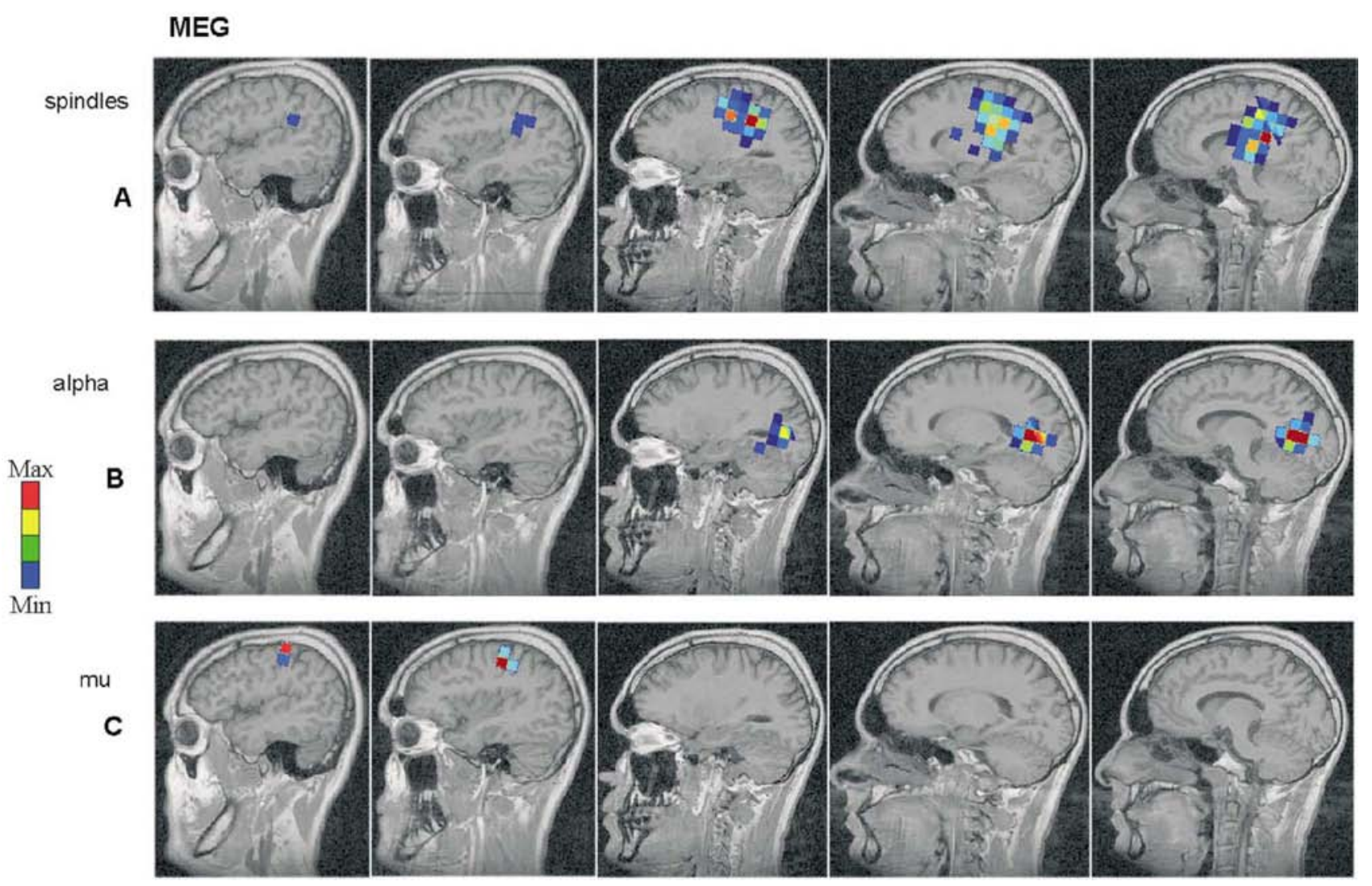

Figure 6. Dipole density plots of the MEG spindles (A), and alpha (B) and mu (C) rhythms of subject 4. Voxels containing a relative high amount of dipoles are represented in red. Blue voxels contain relatively few dipoles. Voxels comprising less than $10 \%$ of the maximal amount of dipoles present in the red voxels are omitted for clarity. These plots demonstrate that there was no overlap of the alpha and mu clusters. Furthermore, the "hot spot" of the alpha rhythm was located more superficially than those of the spindles, whereas the spindle cluster was more widespread than that of the alpha rhythm.

ous rhythmic activities: alpha and mu rhythms and sleep spindles. A practical advantage of the software used in this study is its ability to perform source localization on large amounts of data (as is the case in spontaneous brain rhythms) in a semi-automated and rapid manner. Furthermore, systematic differences between spindles, and alpha and mu rhythms were found with our method. In addition these differences were reproducible over subjects (Figure 5). Together with the dipole density plots these methods provide insight in the spatial distribution and the variability of the dipole solutions.

The conditions that the dipole parameters in the model had to comply with were based on several arguments. First of all, PCA demonstrated that the use of a stationary dipole model with dipolar configuration would need at least 5 simultaneous sources to explain the spindle as a whole adequately. Other groups also reported multiple components of the MEG spindle signal ( $\mathrm{Lu}$ et al., 1992, Shih et al., 2000). However, the use of many dipoles may result in unstable solutions of the model. Figure 3 demonstrates that the contribution of the components of one spindle epoch varied in time, suggesting that individual samples within a spindle can be explained by fewer dipoles. We therefore choose a moving dipole model with only 7 free parameters per time sample, in- stead of a stationary dipole model with 5 times 4 dipole parameters and 5 amplitude parameters per time sample. As both hemispheres generate spindle activity the choice of a dipole in each hemisphere seems plausible. Furthermore, the power distribution of MEG spindles (Figure 2) suggests a source in each hemisphere. Thus the PSD model will be sufficient for time samples in which only two components are simultaneously active. This implies that time samples in which one or two symmetric components dominate the signal are identified. In this way we take into account the stochastic behavior both in time and space of the various principal components of the spindle.

One of the main findings of the present study is that the "center of gravity" of the MEG sleep spindle activity was situated within the centro-parietal region. The "hot spots" were located deep under the cortical surface and the corresponding clusters were rather widespread. These results suggest (see first part discussion) that relatively extended cortical areas in the centro-parietal regions, most likely the whole parietal cortex and the posterior part of the frontal cortex, are involved in the generation of sleep spindles as recorded using the MEG. Interestingly, the "hot spots" of the MEG spindle dipoles appear to coincide with dipoles of MEG slow waves during sleep as described by Simon et al. (2000). This indi- 


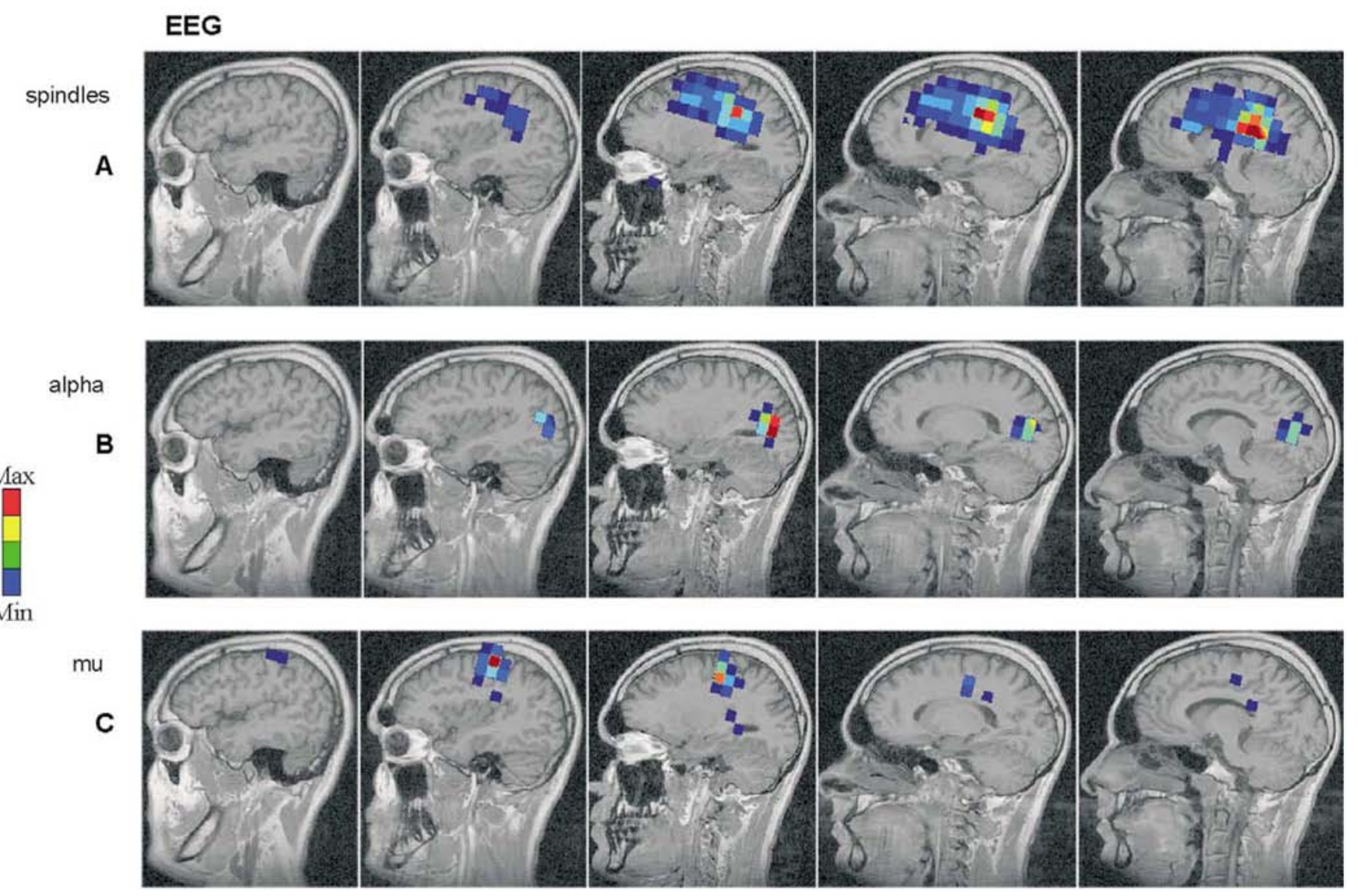

Figure 7. Dipole density plots of the EEG spindles (A), and alpha (B) and mu (C) rhythms of subject 4 . As in the MEG the "hot spots" of the EEG spindles were present in the centro-posterior areas, however, the EEG dipoles spread to more frontal areas. Again there is no overlap of the mu and alpha dipoles.

\section{A}

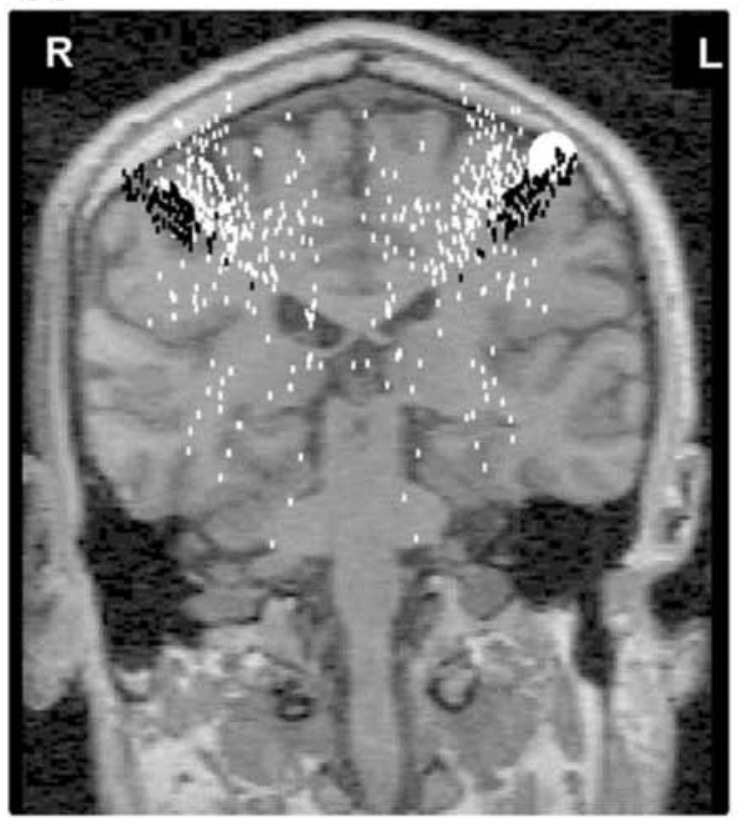

\section{B}

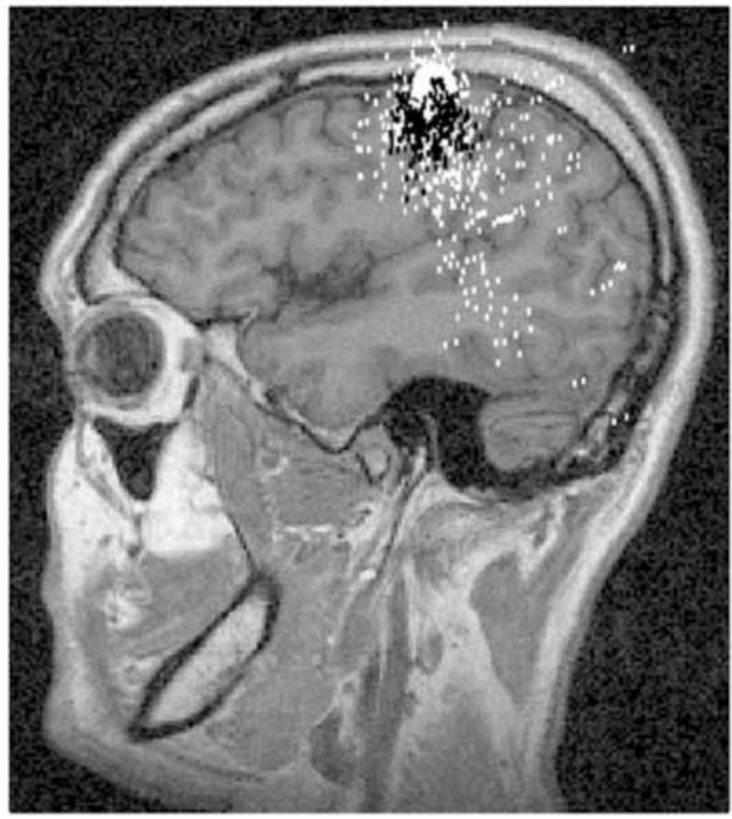

Figure 8. Comparison of EEG (white dots) and MEG (black dots) sources of the mu rhythm with the localization of the SEF (large white dot) after stimulation of the right medial nerve of subject 4 . Plots were made of the MRI slices of the coronal (A) and sagittal (B) which corresponded to the plane of the SEF localization. Mainly dipoles of the MEG mu rhythm are clustered around the central sulcus where the SEF is localized. The dipoles of the EEG mu rhythm are scattered around this area. 
cates that, although the brain produces activity in distinctive frequency bands during sleep, these rhythms may very well originate from the same brain regions. It is unlikely that these deeply located spindle dipoles represent thalamic activity. Sleep spindles are generated in the reticular nucleus of the thalamus, and through thalamo-cortical neurons the cortex is triggered to generate spindle bursts (Steriade et al., 1993). The anatomical organization of the thalamic neurons is such that it forms a closed structure and thus a closed field. The outside of a closed field hardly produces MEG/EEG activity (Lopes da Silva and Van Rotterdam, 1993), especially from a larger distance as is the case in MEG/EEG recordings. It is obvious that the spontaneous oscillations recorded with MEG/ EEG are derived from pyramidal neurons of the cortex (Lopes da Silva and Van Rotterdam, 1993).

The anterior part of the frontal lobe and the occipital lobe do not appear to be involved in the generation of spontaneously occurring MEG sleep spindles. In contrast, Shih et al. (2000) found MEG spindle dipoles in all 4 cerebral lobes, most frequently in the parietal and frontal lobes. However, in that study chloral hydrate was administered to the subjects to induce sleep, which augments the occurrence of low beta frequencies with a maximum in the fronto-central regions (Kozelka and Pedley, 1990). Furthermore, they used a larger frequency band-width $(8-20 \mathrm{~Hz})$ which may include fast wave activity sources in their results. Nevertheless, it appears that MEG spindle generators are not restricted to a small cortical area. A distinct cluster of MEG dipoles in more anterior regions could not be found in any of our subjects, with the possible exception of subject 2 (Figure 5) where the dipoles spread somewhat more anteriorly.

The dipole clusters of the MEG alpha rhythms were located in the region of the parieto-occipital sulcus. This was most clear in subject 4 who displayed an alpha rhythm with large amplitude. Several authors report MEG alpha dipoles predominantly in the parieto-occipital area (Lü et al., 1991, Salmelin and Hari, 1994, Salenius et al., 1995, Hari et al., 1997) which is in line with our results. Additionally some dipole solutions were found around the calcarine sulcus (Williamson and Kaufman, 1989, Salmelin and Hari, 1994, Salenius et al., 1995, Hari et al., 1997, Parra et al., 2000). With intra-cranial recordings alpha rhythms could be measured throughout the occipital lobes of both hemispheres and even outside the occipital lobe (Perez-Borja et al., 1962). Nevertheless, it is evident that the "center of gravity" of MEG alpha activity arises from this region.

When comparing the results of the alpha rhythms and the sleep spindles we should emphasize that there was no overlap of the "centers of gravity" of these two kinds of rhythms. This indicates that different regions of the cortex are involved in the generation of these brain rhythms. The same applies for the mu rhythm that appeared to be generated in a distinct brain region than the alpha rhythm and the sleep spindles. The superficial location of the mu rhythm dipoles (especially in MEG) suggests that the mu rhythm is generated in a relatively well localized cortical area. The close spatial relation of the MEG mu rhythm dipoles with the dipoles of the N20 component of the medial nerve SEF demonstrates that the mu rhythm arises from the cortex around the central sulcus. A closer inspection of the MEG mu rhythm dipoles in the coronal MRI section of subject 4 (Figure 8 ) revealed that the dipoles of the mu rhythm follow the course of the central sulcus. Corticographic studies (Arroyo et al., 1993, Crone et al., 1998) established a relationship between the mu rhythm and sensorimotor activity of the central sulcus, which was confirmed by MEG measurements (Salmelin and Hari, 1994, Hari et al., 1997). The distances of the spindle dipole cluster to the cortex is larger than that of the alpha and mu rhythms. This suggests that a larger part of the cortex in involved in the generation of sleep spindles compared to the alpha and mu rhythms.

In accordance with the MEG, the EEG spindle dipoles of subject 4 form a deep lying cluster in the centroparietal regions with "hot spots" in the same areas as in MEG. In this respect sources of EEG spindles appear to be the same as MEG spindle sources. The posterior MEG and EEG spindle clusters presumably correspond to the sources of the fast spindle peak as can be seen in the power distributions of the fast spindle peaks (Figure 2). This is probably in concordance with the results of Anderer et al. (2001) who demonstrated the involvement of the medial part of the parietal lobe in the generation of fast EEG spindles when analyzed by LORETA. However, this study showed that a major part of the frontal lobe appears to be involved in the generation of slow EEG spindles. As the EEG spectrum of subject 4 demonstrates an anterior distribution of the slow spindle activity it is likely that the dipoles in the anterior brain regions represent such slow spindles. Only subject 4 exhibited a slow spindle peak in the EEG. Accordingly Werth et al. (1997) could not find slow spindles in all subjects. It is noteworthy that the presence of the slow spindle peak in the EEG of subject 4 does not coincide with the occurrence of a slow spindle peak in the MEG. A possible explanation for this finding would be that the orientation of the slow spindle source would be purely radial, and therefore not measurable using the MEG. This is not likely, however, since the power distribution of the slow spindle peak is widespread (Figure 2d, left) and therefore all orientations are expected to be present. The power distribution suggests that an extended cortical area is involved in the generation of slow spindles. Furthermore, we examined whether radially oriented EEG dipoles prevailed in the frontal regions but it appeared that both anterior and posterior regions encompassed equal distributions of radial and tangential oriented dipoles (data not shown). Moreover, Anderer et al. (2001) demonstrated using LORETA that the part of the frontal cortex which is involved in the generation of slow spindles is rather extended, thus it is unlikely that it would not involve both radial and tangential oriented sources. The difference between Anderer's and our results, could be that we used a rather high 
threshold in order to accept equivalent sources as being relevant, while Anderer et al. (2001) performed a statistical group analysis on the differences in LORETA power over spindle and spindle free epochs. Another possibility is that in our moving dipole model we selected only those time points where one source dominates the others, because only time samples accounting for more than $90 \%$ of the variance are included in the analysis. Nevertheless, when we tested relaxing the $90 \%$ criterion, in such a way that the same fraction of dipoles was selected as in the EEG, we did not find a clear spread of MEG sources to more frontal areas (data not shown).

\section{Conclusions}

Source localization of MEG spindles using an equivalent moving dipole model revealed a major cluster of dipoles in the centro-posterior area which suggests involvement of the pre- and post-central areas in the generation of MEG sleep spindles. Furthermore, this study demonstrates that dipoles of the MEG spindles, and alpha and mu rhythms display different spatial distributions. This emphasizes the perspective of various cortical regions that oscillate within the same frequency band but have different spatial organizations and different functional aspects.

Acknowledgments - We thank Professor Dr. C. J. Stam for reviewing the manuscript.

\section{References}

Anderer P, Klo“sch G, Gruber G, Trenker E, Pascual-Marqui RD, Zeitlhofer J, Barbanol MJ, Rappelsberger P, Saletu B. Low-resolution brain electromagnetic tomography revealed simultaneously active frontal and parietal sleep spindle sources in the human cortex. Neuroscience 2001;103(3):581-592.

Arroyo S, Lesser RP, Gordon B, Uematsu S, Jackson D, Webber R. Functional significance of the mu rhythm of human cortex: an electrophysiologic study with subdural electrodes. Electroenceph clin Neurophysiol 1993;87:76-87.

Caderas M, Niedermeyer E, Uematsu S, Long DM, Nastalski J. Sleep spindles recorded from deep cerebral structures in man. Clin Electroencephalogr 1982;13(4):216-225.

Crone NE, Miglioretti DL, Gordon B, Sieracki JM, Wilson MT, Uematsu S, Lesser RP. Functional mapping of human sensorimotor cortex with electrocorticographic spectral analysis. I. Alpha and beta event-related desynchronization. Brain 1998;121:2271-2299.

De Munck JC, Verbunt JPA, Van 't Ent D, Van Dijk BW. The use of an MEG device as 3D digitizer and motion monitoring system. Phys Med Biol 2001a;46:2041-2052.

De Munck JC, De Jongh A, Van Dijk BW. The localization of spontaneous brain activity: an efficient way to analyze large data sets. IEEE Trans Biomed Eng 2001b;48:1221-1228.

Hari R, Salmelin R, Mäkelä JP, Salenius S, Helle M. Magnetoencephalographic cortical rhythms. Int J Psychobiol 1997;26:51-62.

Hoogeman MS, Van Herk M, Zijp L, Meinders J, Muller P, Koper PCM, Boersma LJ, Lebesque JV. The design and implementation of a multicenter volumetric and dosimetric database (Extended Abstract) 13th ICCR. Heidelberg, Germany, 2000. p. 82-84.
Hughes JR, Hendrix DE, Cohen J, Duffy FH, Mayman CI, Scholl ML, Cuffin BN. Relationship of the magnetoecephalogram to the electroencephalogram. Normal wake and sleep activity. Electroenceph clin Neurophysiol 1976;40:261-278.

Kozelka JW, Pedley TA. Beta and mu rhythms. J Clin Neurophysiol 1990;7(2):191-207.

Lopes da Silva FH, Van Rotterdam A. Biophysical aspects of EEG and magnetoencephalogram generation. In: Niedermeyer E, Lopes da Silva FH, editors. Electroencephalography. Basic principles, clinical applications and related fields, 3rd ed. Baltimore, MD: Williams and Wilkins, 1993. pp. 78-91.

Lu S-T, Kajola M, Joutsiniemi SL, Knuutila J, Hari R. Generator sites of spontaneous MEG activity during sleep. Electroenceph clin Neurophysiol 1992;82:182-196.

Lu“ Z-L, Wang J-Z, Williamson S. Neuronal sources of human parietooccipital alpha rhythm. In: Hoke M, Erne' SN, Okada YC, Romani GL, editors. Neuronal sources of human parieto-occipital alpha rhythm, Biomagnetism. Clinical aspects, Amsterdam: Excerpta Medica, 1991. pp. 33-37.

Nakasato N, Kado H, Nakanishi M, Koyanagi M, Kasai N, Niizuma H, Yoshimoto T. Magnetic detection of sleep spindles in normal subjects. Electroenceph clin Neurophysiol 1990;76:123-130.

Parra J, Meeren HKM, Kalitzin S, Suffczynski P, De Munck JC, Kasteleijn- Nolst Trenite' DGA, Lopes da Silva FH. Magnetic source imaging in fixation-off sensitivity: relationship with alpha rhythm. J Clin Neurophysiol 2000;17(2):212-223.

Perez-Borja C, Chatrian GE, Tyce FA, RiversMH. Electrographic patterns of the occipital lobe in man: a topographic study based on use of implanted electrodes. Electroenceph clin Neurophysiol 1962;14:171-182.

Salenius S, Kajola M, Thompson WL, Kosslyn S, Hari R. Reactivity of magnetic parieto-occipital alpha rhythm during visual imagery. Electroenceph clin Neurophysiol 1995;95:453-462.

Salmelin R, Hari R. Characterization of spontaneous MEG rhythms in healthy adults. Electroenceph clin Neurophysiol 1994;91:237-248.

Shih JJ, Weisend MP, Davis JT, Huang M. Magnetoencephalographic characterization of sleep spindles in humans. J Clin Neurophysiol 2000;17(2):224-231.

Simon NR, Manshanden I, Lopes da Silva FH. A MEG study of sleep. Brain Res 2000;860:64-76.

Steriade M, McCormick DA, Sejnowski TJ. Thalamocortical oscillations in the sleeping and aroused brain. Science 1993;262:679-685.

Tiihonen J, Kajola M, Hari R. Magnetic mu rhythm in man. Neuroscience 1989;32:793-800.

Uchida S, Hirai N, Nakabayashi T, Maehara T, Shimizu H. Sleep spindle in human orbitofrontal cortex. Sleep Res Online 1999;2:96.

Van Herk M, de Jaeger K, De Munck J, Hoogeman M, Meinders J, Ploeger L, Zijp L. A delineation system for N modalities - software aspects (Extended Abstract) 13th ICCR. Heidelberg, Germany, 2000. p. 73-75.

Werth E, Achermann P, Dijk DJ, Borbe'ly A. Spindle frequency activity in the sleep EEG: individual differences and topographic distribution. Electroenceph clin Neurophysiol 1997;103:535-542.

Williamson S, Kaufman L. Advances in neuromagnetic instrumentation and studies of spontaneous brain activity. Brain Topogr 1989;2:129-139.

Yoshida H, Iramina K, Ueno S. Source models of sleep spindles using MEG and EEG measurements. Brain Topogr 1996;8(3):303-307. 